\title{
Sensitivities for a Single Drop Simulation*
}

\author{
Christian H. Bischof, H. Martin Bücker, Arno Rasch, and Emil Slusanschi \\ Institute for Scientific Computing, Aachen University, D-52056 Aachen, Germany \\ \{bischof, buecker, rasch, slusanschi\}@sc.rwth-aachen.de \\ http://www.sc.rwth-aachen.de
}

\begin{abstract}
In process engineering, a single drop is investigated to better understand its physical and chemical behavior. Laboratory experiments using the Nuclear Magnetic Resonance (NMR) technology are prepared by numerical simulations aiming at finding a suitable geometry of the measuring cell. In the underlying numerical optimization problem, derivatives of the flow field around a single drop with respect to geometric parameters are needed. Rather than using numerical differentiation based on divided differencing, a technique called automatic differentiation is used to compute truncation-error free derivative values. It is shown that automatic differentiation is comparable to numerical differentiation in terms of CPU time but eliminates potential problems in accuracy encountered when using numerical differentiation.
\end{abstract}

\section{Introduction}

In an interdisciplinary project, process engineers and computer scientists are aiming at properly designing the geometry of the measuring cell by numerical simulations. In the actual laboratory experiments, one is trying to fix a single drop at a certain position in a liquid in order to analyze fluid-fluid interactions by using the Nuclear Magnetic Resonance (NMR) technology. The goal is to avoid spatial movement of the single drop enabling appropriate NMR measurements. From a conceptual point of view the numerical simulation is minimizing a suitable objective function representing the position of a single drop in the flow field with respect to parameters characterizing the geometry of the underlying computational domain. A crucial ingredient of most numerical optimization algorithms is the derivative of the objective function with respect to the free variables. In the present situation, the objective function is given by a largescale computational fluid dynamics code that, given the geometric parameters of the computational domain, computes the flow field by solving the NavierStokes equations and some additional post-processing of the flow field which we neglect throughout this note for the sake of simplicity.

One option to evaluate the derivatives of the flow field with respect to geometric parameters consists of a numerical approach based on divided differencing.

\footnotetext{
* This research is partially supported by the Deutsche Forschungsgemeinschaft (DFG) within SFB 540 "Model-based experimental analysis of kinetic phenomena in fluid multi-phase reactive systems," Aachen University, Germany.
} 
The advantage of divided differencing is its simplicity: approximations to derivatives of certain accuracy are available by evaluating the function whose derivatives are sought in a black box fashion at suitably perturbed input arguments. However, there is always truncation error and, when the input arguments differ only slightly, the resulting cancellation error may also dramatically influence the accuracy of the derivative approximations.

In this note, we apply a truncation error-free technique called automatic differentiation to obtain derivatives of the flow field with respect to geometric parameters. In automatic differentiation (AD), a given computer program to evaluate some function is transformed into another program capable of evaluating the original function along with its derivatives. More precisely, we apply the AD system ADIFOR [2] to a general purpose finite element package SEPRAN [14] written in Fortran 77. In doing so, we extend previous work [3] where the flow solver module of SEPRAN was transformed by ADIFOR to evaluate derivatives of the flow field with respect to flow parameters such as the Reynolds number or the angle of attack. Now, we additionally apply AD to SEPRAN's grid generator enabling derivatives with respect to geometric parameters. A similar approach in the area of aerodynamics is studied in 6] where AD is applied to the WTCO wing grid generator and the TLNS3D Navier-Stokes flow solver, both Fortran 77 programs. Details on developing an AD-enhanced version of TLNS3D are given in [10,9]. Another application of AD applied to a grid generator called CSCMDO which is written in $\mathrm{C}$ is described in 4. Aerodynamic sensitivities with respect to geometric parameters are also reported in [8].

The structure of this note is as follows. In Sec. 2, the basic idea behind automatic differentiation is briefly summarized and issues of applying AD to SEPRAN are sketched. The simulation of a single drop using SEPRAN and the corresponding derivatives obtained from AD are given in Sec.3.

\section{Automatic Differentiation and the SEPRAN Package}

When a function is given in the form of a computer code, AD may be used to generate another computer program capable of evaluating the function and its derivatives. The basic idea of $\mathrm{AD}$ is to treat a computer program as the composition of a large sequence of elementary functions and intrinsics whose derivatives are known. Then, these derivatives are accumulated using the chain rule. When using an $\mathrm{AD}$ tool a user has to specify the output variables whose derivatives are sought, called dependent variables, and the input variables with respect to which one is differentiating, called independent variables. A variable is called active if its value depends on the value of an independent variable and it influences the value of a dependent variable. More details on AD are given in [11, $13,7,12,1$. The AD technology is not only applicable to small computer codes but also scales to large programs of several hundred thousand lines of code.

In this note, we apply the AD tool ADIFOR [2] to the general purpose finite element package SEPRAN [14, developed at "Ingenieursbureau SEPRA" and Delft University of Technology. The SEPRAN package solves the continuity and Navier-Stokes equations. SEPRAN is employed in a wide variety of engineering applications [5 15,16,18 17 19,20] including laminar or turbulent flow of 


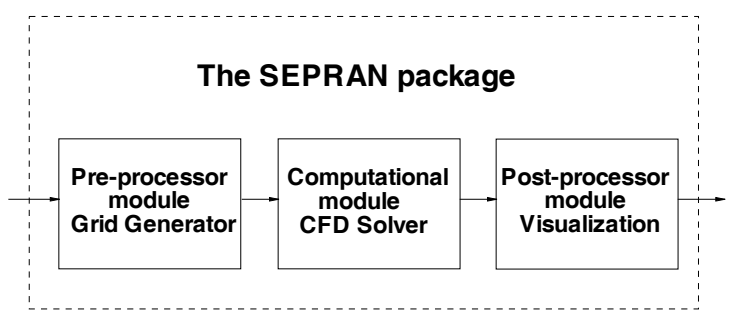

Fig. 1. The structure of the finite element package SEPRAN

incompressible liquids. The SEPRAN package consists of about 800,000 lines of Fortran 77 in several modules, such as the pre-processing (grid generation), computational and post-processing modules schematically depicted in Fig. 1 In a previous work [3], we successfully applied AD to the computational module where approximately 400,000 lines were automatically transformed. In this note, we not only apply AD to the CFD solver but also to the grid generation part consisting of 130,000 additional lines of code in order to get derivatives for the single drop problem to be described in Sec.3.

In order to be able to use the ADIFOR tool to obtain the augmented version of the code, SEPRAN.AD, a preprocessing step has to be taken. This comprises of massaging the SEPRAN source code so that it adheres to the Fortran77 language standard. It is worth noticing that SEPRAN is generally a "clean" code, which requires little changes by hand in the original code in order to be able to apply ADIFOR to it. However, some problems, like type mismatches and calling of some subroutines with different number of arguments were encountered. Usually, normal compilers do not detect such problems because they are examining only one Fortran file at a time, whereas ADIFOR does a global data flow and dependency analysis and therefore is able to detect these inconsistencies. More details are given in [3]. A problem that was a bit harder to detect was encountered in the grid generation part of the SEPRAN code, and it has to do with a certain way of referencing memory in Fortran through the use of "read" and "write" instructions. When the ADIFOR tool encounters an active variable in a statement that does a "read" or a "write", it generates a warning message. In this particular case, a value was written to memory using "write" and was later used by calling a corresponding "read." Therefore, any AD tool is unable to recognize whether or not this value has changed. In order to maintain the "logic link" between the dependent and independent variables, a common block was inserted instead of the mentioned use of the "read/write" instructions. In this way the augmented code SEPRAN.AD, consisting of the grid generation and computational part totaling about 703,000 lines of code, produced correct results as presented in the following section. Note that the code massaging part, which requires some manual work is done only once in the developing cycle of the augmented version of SEPRAN. 


\section{Sensitivities of a Single Drop}

The study of kinetic phenomena in a single drop is the topic of an interdisciplinary project at Aachen University within the Collaborative Research Center SFB 540. To this end a two-dimensional numerical simulation of a measuring cell is implemented using SEPRAN. This simulation is stationary and rotationally symmetrical under the assumption of Newtonian and incompressible fluids with a non-deformable drop. The goal of the project is to enable the measurements of the velocity and material properties of a single drop using the NMR technology. The design of the measuring cell has to be optimized so that the drop remains stationary for a certain period of time during which the measurements are taking place. The idea is to maintain the drop stable using a counter current flow. The drop is initiated at the bottom of the measuring cell with a special pump and it keeps on rising, until its velocity and that of the counter current flow are the same, and in this way the drop reaches its desired stable position and the NMR measurements can then take place. After the measurements, the counter current flow is stopped and the drop rises until it reaches a collector where it is absorbed. Given the velocity of the inflow $v_{0}$, the density $\rho$, and viscosity $\mu$ of the two phases (drop and counter current), the SEPRAN package is able to compute the velocity and pressure fields at any point in the measuring cell. The design of this measuring cell depends on the following geometric parameters schematically depicted in Fig. 2. The first parameter, $\alpha$, is the angle between the vertical axis and the wall of the measuring equipment. The second parameter, $r_{1}$, denotes the radius of the inlet and the third parameter, $r_{2}$, specifies the smallest possible radius of the measuring cell, representing the distance between the symmetric axis and the wall.

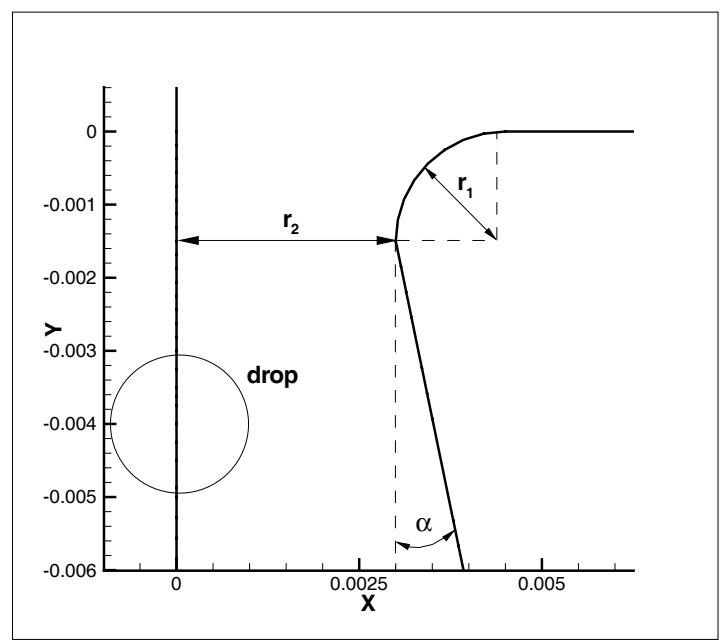

Fig. 2. Geometry of the measuring cell 


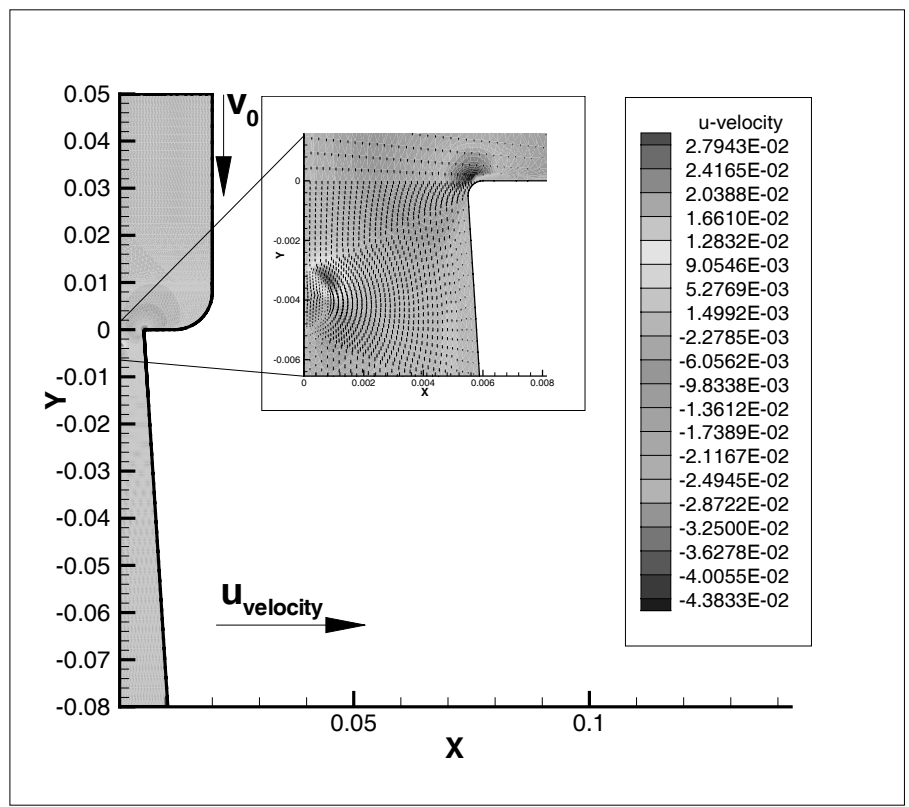

Fig. 3. Original function: horizontal component $u$ of the velocity

From an abstract point of view, the computer program implements a function $f\left(v_{0}, \rho, \mu, \alpha, r_{1}, r_{2}\right)$ representing the flow field. For the sake of simplicity, we will use $f$ to denote the velocity of the fluid. Note that the actual simulation also computes the pressure field for which a sensitivity analysis can be carried out similar to the one for the velocity given in the following discussion. The velocity field computed by SEPRAN for the flow parameters

$$
v_{0}=-4.2 \cdot 10^{-3}, \quad \rho=1.0 \cdot 10^{3}, \quad \mu=1.0 \cdot 10^{-3},
$$

and the geometric parameters

$$
\alpha=3.6411, \quad r_{1}=5.0 \cdot 10^{-4}, \quad r_{2}=5.5 \cdot 10^{-3}
$$

is given in Fig. 3. More precisely, the figure shows the horizontal component, $u$, of the velocity field with a zoom in the region where the drop is kept stable. The vectors of the flow field are also presented in this zoom.

In a numerical optimization algorithm, the derivatives of the velocity field with respect to the set of design parameters (1) are needed. We use automatic differentiation for the computation of these derivatives, $\partial f / \partial \alpha, \partial f / \partial r_{1}$ and $\partial f / \partial r_{2}$. As an illustrating example, the results presented in Fig. 4 show the derivatives of the horizontal component of the velocity with respect to the angle $\alpha$. The figure demonstrates that the largest increase of this velocity component with respect to changes in $\alpha$ occurs in the vicinity of the drop. 


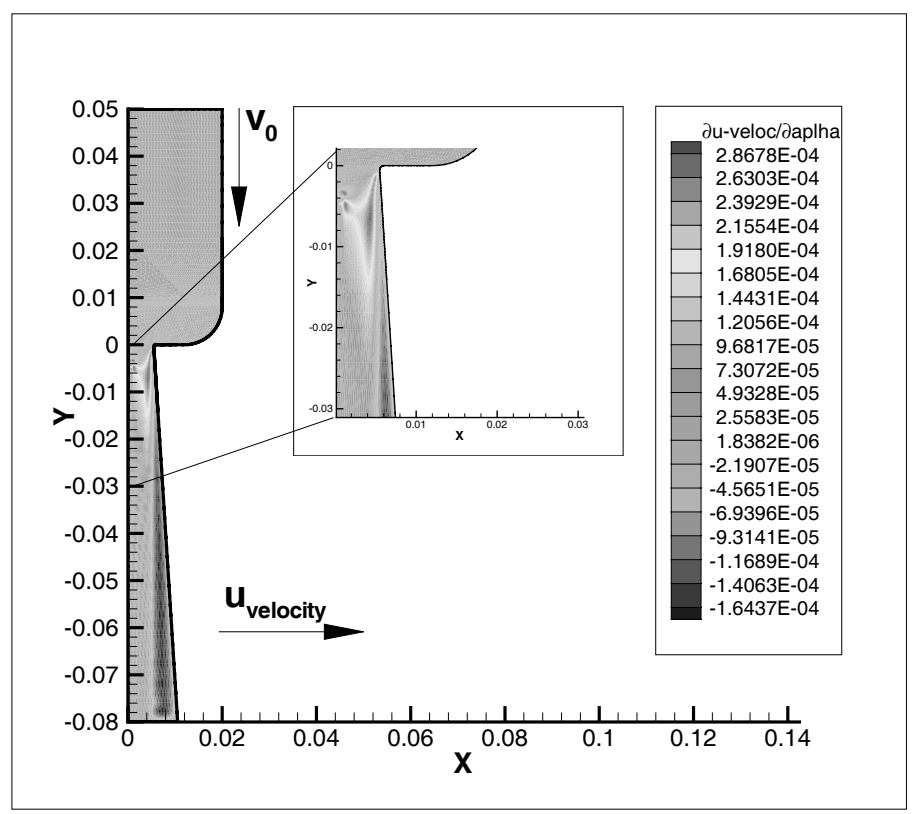

Fig. 4. AD-generated derivative $\partial u / \partial \alpha$ field

To illustrate the advantage of the $\mathrm{AD}$ approach in terms of accuracy, we present a comparison with the traditional numerical approach of computing derivatives making use of divided differences. We will use DD as a shorthand notation for first-order forward divided differences, defined by

$$
\frac{\partial f\left(v_{0}, \rho, \mu, \alpha, r_{1}, r_{2}\right)}{\partial \alpha} \approx \frac{f\left(v_{0}, \rho, \mu, \alpha+h, r_{1}, r_{2}\right)-f\left(v_{0}, \rho, \mu, \alpha, r_{1}, r_{2}\right)}{h}
$$

where $h$ is a suitable chosen step size for the approximation. Equation (2) can also be written for the other geometric parameters, $r_{1}$ and $r_{2}$. DD is easy to implement in a black-box manner. On the other hand though, the accuracy of the approximations provided depends very much on choosing a suitable step size $h$. The problem with DD is that, in general, there is no a priori knowledge of a suitable step size which may also be hard to determine. Furthermore, there are cancellation and truncation errors that the user has to contend with, all contributing to unavoidable errors on the end result. When employing automatic differentiation, however, there is no truncation error to contend with, the results are accurate up to machine precision and there is no need to experiment with a step size.

For the derivative $\partial u / \partial \alpha$, it turns out that it is possible to find a suitable step size $h=10^{-3}$ where the infinity norm of the difference between the DD and AD-generated derivatives is $1.1 \cdot 10^{-7}$. However, when considering the derivative $\partial u / \partial r_{2}$, it is no longer possible to find an accurate DD-approximation. After 


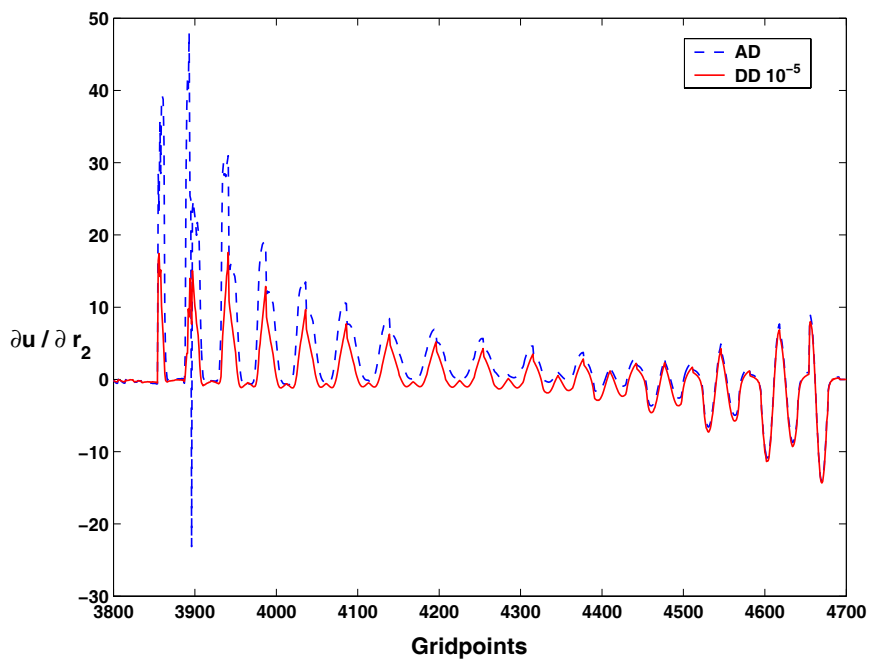

Fig. 5. AD-DD comparison of the derivative of the $\partial u / \partial r_{2}$

identifying a suitable step size of $h=10^{-5}$, the corresponding infinity norm is 38.94 representing a large difference between the values computed by AD and DD. An explanation is given by Fig. 5 showing the derivative $\partial u / \partial r_{2}$ versus a subset of selected grid points. The grid points shown in this figure are numbered from 3800 to 4700 and are located in the inlet region near the drop. At certain grid points, the divided difference approximations are not able to give us the exact derivatives computed by AD. A similar analysis holds for the derivative $\partial u / \partial r_{1}$ where the infinity norm of the difference between AD and DD is 39.32.

Therefore we conclude that using a numerical approach to calculating the required sensitivities in order to optimize the geometry of the measuring cell is not appropriate, as these approximations may be inaccurate. In contrast, automatic differentiation provides reliable derivative values. Moreover, considering that an optimization must be conducted with respect to a series of geometry parameters $\left(\alpha, r_{1}, r_{2}\right)$ it is not advantageous to have different suitable step sizes, namely $10^{-3}$ and $10^{-5}$, for different derivatives.

When it comes to analyzing the performance of the AD augmented code compared to the original simulation, we observe an increase in execution time by a factor of 4.15 and an increase in memory usage by a factor of 3.41 . The simulations were carried out on a $1733 \mathrm{MHz}$ Pentium 4 Linux machine with 2 GB of main memory. The number of independent variables in this simulation is three, the angle $\alpha$, and the two radii $r_{1}$ and $r_{2}$. Therefore the expected increase in memory requirement is within normal limits. Concerning the execution times, the approximations obtained by forward divided differences for three partial derivatives require at least four evaluations of the original function, provided a "suitable" step size is already known. When employing AD, on the other hand, the expected increase in execution time is proportional to the number 
of independent variables specified. Therefore, the observed ratio of 4.15 in the increase in execution time, can be considered very good, as the results are reliable and precise up to machine precision.

\section{Conclusions}

Laboratory experiments for the analysis of fluid-fluid interactions are prepared by numerical simulations. In particular, the geometry of the underlying measuring cell is designed in order to keep a constant position of a single drop in a liquid. The SEPRAN package is used to simulate the flow field for given geometric parameters. The ADIFOR system is applied to obtain derivatives of the flow field with respect to geometric parameters. This approach using automatic differentiation is compared to a numerical approach based on divided differencing. The major advantage of automatic differentiation in this application is the availability of derivative values without truncation error. Moreover, time complexity is comparable to that of a first-order forward divided differencing approach.

Currently, we are trying to determine a suitable objective function for the future optimization of the design of the measuring cell. However, from the point of view of algorithmic ingredients, the major computational challenge is to efficiently and accurately evaluate the derivatives of the flow field with respect to the geometric parameters as described in this work. An implementation of the objective function will consist of only a few lines of code compared to the complete SEPRAN package and its remaining differentiation will easily be accomplished by the application of an AD tool.

Acknowledgments. The authors would like to thank the collaborators at the Institute of Thermal Unit Operations, Aachen University. In particular, Edwin Groß-Hardt deserves special recognition for making available the grid and problem specifications of the underlying problem used in the SEPRAN simulation. We would also like to thank Jakob Risch for his notable contribution during the initial phase of applying ADIFOR to SEPRAN. This research is partially supported by the Deutsche Forschungsgemeinschaft (DFG) within SFB 540 "Modelbased experimental analysis of kinetic phenomena in fluid multi-phase reactive systems," Aachen University, Germany.

\section{References}

[1] M. Berz, C. Bischof, G. Corliss, and A. Griewank. Computational Differentiation: Techniques, Applications, and Tools. SIAM, Philadelphia, 1996.

[2] C. Bischof, A. Carle, P. Khademi, and A. Mauer. ADIFOR 2.0: Automatic differentiation of Fortran 77 programs. IEEE Computational Science $\&$ Engineering, 3(3):18-32, 1996.

[3] C. H. Bischof, H. M. Bücker, B. Lang, A. Rasch, and J. W. Risch. On the Use of a Differentiated Finite Element Package for Sensitivity Analysis. In V. N. Alexandrov, J. J. Dongarra, B. A. Juliano, R. S. Renner, and C. J. K. Tan, editors, Computational Science - ICCS 2001, Proceedings of the International Conference on Computational Science, San Francisco, USA, May 28-30, 2001. Part I, volume 2073 of Lecture Notes in Computer Science, pages 795-801, Berlin, 2001. Springer. 
[4] C. H. Bischof, A. Mauer, W. T. Jones, and J. Samareh. Experiences with automatic differentiation applied to a volume grid generation code. Journal of Aircraft, 35(4):569-573, 1998.

[5] E. G. T. Bosch and C. J. M. Lasance. High accuracy thermal interface resistance measurement using a transient method. Electronics Cooling Magazine, 6(3), 2000.

[6] A. Carle, L. L. Green, C. H. Bischof, and P. A. Newman. Applications of automatic differentiation in CFD. In Proceedings of the 25th AIAA Fluid Dynamics Conference, Colorado Springs, CO, USA, June 20-23, 1994, AIAA Paper 94-2197, 1994.

[7] G. Corliss, C. Faure, A. Griewank, L. Hascoët, and U. Naumann, editors. Automatic Differentiation of Algorithms: From Simulation to Optimization. Springer, New York, 2002.

[8] S. A. Forth and T. P. Evans. Aerofoil optimisation via AD of a multigrid cellvertex Euler flow solver. In Corliss et al. 7], chapter 17, pages 153-160.

[9] L. Green, P. Newman, and K. Haigler. Sensitivity derivatives for advanced CFD algorithm and viscous modeling parameters via automatic differentiation. In Proceedings of the 11th AIAA Computational Fluid Dynamics Conference, Orlando, FL, USA, July 6-9, 1993, AIAA Paper 93-3321, 1993.

[10] L. L. Green, P. A. Newman, and K. J. Haigler. Sensitivity derivatives for advanced CFD algorithm and viscous modeling parameters via automatic differentiation. Journal of Computational Physics, 125(2):313-324, 1996.

[11] A. Griewank. Evaluating Derivatives: Principles and Techniques of Algorithmic Differentiation. SIAM, Philadelphia, 2000.

[12] A. Griewank and G. Corliss. Automatic Differentiation of Algorithms. SIAM, Philadelphia, 1991.

[13] L. B. Rall. Automatic Differentiation: Techniques and Applications, volume 120 of Lecture Notes in Computer Science. Springer-Verlag, Berlin, 1981.

[14] G. Segal. SEPRAN Users Manual. Ingenieursbureau Sepra, Leidschendam, NL, 1993.

[15] G. Segal, C. Vuik, and F. Vermolen. A conserving discretization for the free boundary in a two-dimensional Stefan problem. Journal of Computational Physics, 141(1):1-21, 1998.

[16] A. P. van den Berg, P. E. van Keken, and D. A. Yuen. The effects of a composite non-Newtonian and Newtonian rheology on mantle convection. Geophys. J. Int., 115:62-78, 1993.

[17] P. E. van Keken, C. J. Spiers, A. P. van den Berg, and E. J. Muyzert. The effective viscosity of rocksalt: implementation of steady-state creep laws in numerical models of salt diapirism. Tectonophysics, 225:457-476, 1993.

[18] P. E. van Keken, D. A. Yuen, and L. R. Petzold. DASPK: a new high order and adaptive time-integration technique with applications to mantle convection with strongly temperature- and pressure-dependent rheology. Geophysical $\&$ Astrophysical Fluid Dynamics, 80:57-74, 1995.

[19] N. J. Vlaar, P. E. van Keken, and A. P. van den Berg. Cooling of the Earth in the Archaean: consequences of pressure-release melting in a hot mantle. Earth Plan. Sci. Lett., 121:1-18, 1994.

[20] C. Vuik, A. Segal, and F. J. Vermolen. A conserving discretization for a Stefan problem with an interface reaction at the free boundary. Computing and Visualization in Science, 3(1/2):109-114, 2000. 